\title{
DYNAMIC POLARIZATION IN RADIATION RESISTANT MATERIALS*
}

\author{
RICHARD C. FERNOW ${ }^{\dagger}$ \\ Randall Laboratory of Physics, University of Michigan, Ann Arbor, Michigan 48109, U.S.A.
}

Received 17 August 1978

\begin{abstract}
Measurements of spin effects in small cross section processes have shown the need for more radiation resistant polarized target materials. We have examined the dynamic polarization of a number of substances which are known to be highly resistant to the formation of color centers with particular emphasis on toluene. We have also examined the relation between the maximum polarization and characteristic radiation flux constant to obtain a figure of merit for new materials.
\end{abstract}

Interesting spin effects have recently been observed ${ }^{\prime}$ ) in proton-proton elastic scattering near $90^{\circ}$ in the center of mass. The very small differential cross sections involved require the use of high intensity beams. A serious difficulty with the use of a polarized target in this experiment was the loss of polarization resulting from radiation damage to the target material ${ }^{2}$ ). The polarization $P$ of the target decays exponentially with accumulated radiation flux $\Phi$ through the relation

$P=P_{0} \exp \left(-\Phi / \Phi_{\mathrm{A}}\right)$,

where $P_{0}$ is the initial polarization and $\Phi_{\mathrm{A}}$ is the characteristic flux for the polarization to decay to $1 / \mathrm{e}$ of its initial value. The materials which are used most often today, ethanediol, propanediol, and n-butanol, have $\Phi_{\mathrm{A}}$ values of $(1-4) \times 10^{14}$ particles $/ \mathrm{cm}^{2}$. When used in an intense beam of a few $10^{10}$ particles/s these materials require frequent annealling and replacement every 1-2 days because of non-anneallable radiation damage. It is clearly desirable to find polarized target materials with $\Phi_{\mathrm{A}} \gtrsim 10^{15}$ particles $/ \mathrm{cm}^{2}$.

Studies of radiation yields ${ }^{3,4}$ ) and production of color centers ${ }^{5}$ ) have shown that a number of materials have higher resistance to radiation than the alcohols and diols. In particular aromatic compounds are known to have small radiation yields and good resistance to producing color centers. We have examined a number of these materials in an attempt to find one that would also give polarizations comparable with the $70-80 \%$ typically found with the diols and butanol in ${ }^{3} \mathrm{He}$ evaporation cryostats. In this we have not been successful. However if the beam intensity is high enough some of these materials should be preferable to the standard targets as we discuss below.

* Work supported in part by the U.S. Department of Energy.

+ Present address: Physics Dept., Brookhaven National Laboratory, Upton, L.I., N.Y. 11973, U.S.A.
The materials used in this study were selected from a list of organic materials examined for the production of color centers by Alger et al. ${ }^{5}$ ). These authors assigned each material a relative coloring ability (RCA), equal to the amount of time the material was subjected to an intense electron beam in order to produce a given amount of coloring. The materials varied in RCA from 2 for methanol to $>2000$ for benzene, toluene, and t-butanol. The common polarized target materials ethanediol and n-butanol had RCAs of 8 and 24 respectively. Thus if a suitable material could be found among the top rated materials it could potentially have several orders of magnitude better radiation resistance than the substances currently in use.

We demanded that the materials have suitable physical characteristics for a polarized target. First, the compound was required to be a liquid at room temperature. This is not a necessary requirement but solids present additional complications such as the type and concentration of a solvent. Second, the melting point was required to be greater than $-115^{\circ} \mathrm{C}$. If the melting point is much less than this there is a large probability of melting the sample during the polarized target cooldown. Third, the materials must be able to form stable spheres under liquid nitrogen. All the results presented here are from spheres $1-2 \mathrm{~mm}$ in diameter. Unfortunately two of the top rated materials, benzene and t-butanol, tended to crystallize and disintegrate after freezing and were dropped from further consideration. The six materials with the highest RCAs that met these requirements are listed in table 1.

The problem then becomes to find a free radical which is soluble in the given material and to determine the concentration of the radical which gives the maximum polarization. The stable free radicals which we used in this study are listed in 
TABLE 1

Summary of polarization results. LS: low solubility ${ }^{6}$ ).

\begin{tabular}{|c|c|c|c|c|c|c|c|}
\hline Material & Formula & $\mathrm{RCA}$ & DPPH & DTBN & $\mathrm{Cr}^{v}$ & HTMPO & PX \\
\hline toluene & $-\mathrm{CH}_{3}$ & $>2000$ & 49 & $\left.57^{7}\right)$ & LS & $\sim 2$ & LS \\
\hline n-butyl ether & $\mathrm{C}_{4} \mathrm{H}_{9}-\mathrm{O}-\mathrm{C}_{4} \mathrm{H}_{9}$ & 1000 & $\sim 44$ & $\sim 40$ & LS & 11 & LS \\
\hline ethyl formate & $\begin{aligned} & \mathrm{H}-\mathrm{C}=\mathrm{O} \\
& \mathrm{l} \\
& \mathrm{O}-\mathrm{C}_{2} \mathrm{H}_{5}\end{aligned}$ & 1000 & 38 & $\sim 3$ & LS & - & LS \\
\hline n-pentanol & $\mathrm{CH}_{3}\left(\mathrm{CH}_{2}\right)_{4} \mathrm{OH}$ & 500 & LS & 45 & 62 & - & $\left.57^{8}\right)$ \\
\hline amyl acetate & $\begin{aligned} \mathrm{CH}_{3}-\mathrm{C} & =\mathrm{O} \\
\mathrm{I} & -\mathrm{C}_{5} \mathrm{H}_{11}\end{aligned}$ & 400 & $\sim 45$ & 43 & LS & - & LS \\
\hline benzyl alcohol & $-\mathrm{CH}_{2} \mathrm{OH}$ & 250 & $\sim 10$ & 47 & - & - & LS \\
\hline
\end{tabular}

table 2. The five radicals were DPPH: $2,2-$ Diphenyl-1-picrylhydrazl; DTBN: Di-tert-butylnitroxide; HMBA-Cr ${ }^{v}$ : Sodium bis (2-hydroxy-2methylbutyrato) oxochromate (V) monohydrate ${ }^{9}$ ); HTMPO: 4-Hydroxy-2,2,6,6-tetramethylpiperdino1-oxy; PX: porphyrexide. We also list in table 2 the molecular weight, microwave frequencies corresponding to maximum dynamic polarization for positive and negative enhancements in a $2.508 \mathrm{~T}$ field, and the effective $g$ factor for each radical.

We have done a careful study of the dependence of the polarization on concentration in the top rated material, toluene. The results, shown in fig. 1, show that the polarization at $0.5 \mathrm{~K}$ using DPPH peaked at $50 \%$ for $1 \%$ DPPH by weight. The polarization with DTBN increased with decreasing concentration reaching $62 \%$ for
$0.3 \%$ DTBN. Unfortunately the relaxation time at $0.5 \mathrm{~K}$ was over $5 \mathrm{~h}$ for this concentration. This long relaxation time means that target reversals and thermal calibration measurements would take much longer than normal, making $0.5 \%$ the lowest practical concentration. The radical HTMPO gave very small polarizations while $\mathrm{PX}$ and $\mathrm{HMBA}-\mathrm{Cr}^{\vee}$ were insoluble in toluene. A summary of the results for the maximum practical polarizations obtained at $0.5 \mathrm{~K}$ in the pure substances is given in table 1.

More detailed information on the individual trials of these and some closely related materials is given in table 3 . We have listed the concentration of the radical in percent by weight, whether or not the radical was mixed into the solvent under a vacuum (to remove dissolved oxygen), the maxi-

TABLE 2

Properties of the free radicals.

\begin{tabular}{|c|c|c|c|c|c|}
\hline Radical & Formula & MW & $\begin{array}{c}f^{+} \\
(\mathrm{GHz})\end{array}$ & $\stackrel{f}{(\mathrm{GHz})}$ & $g_{\text {eff }}$ \\
\hline $\mathrm{DPPH}$ & $\left(\mathrm{NO}_{2}\right)_{3} \mathrm{C}_{6} \mathrm{H}_{2} \mathrm{~N}(.) \mathrm{N}\left(\mathrm{C}_{6} \mathrm{H}_{5}\right)_{2}$ & 395 & 70.27 & 70.47 & 2.004 \\
\hline DTBN & $\left(\left(\mathrm{CH}_{3}\right)_{3} \mathrm{C}_{2} \mathrm{NO} ..\right)$ & 144 & 70.27 & 70.52 & 2.005 \\
\hline PX & CNHNHCNHC(CH$)_{2}$ NO(.) & 141 & 70.15 & 70.58 & 2.000 \\
\hline HTMPO & 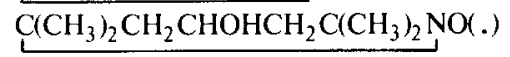 & 172 & 70.25 & 70.58 & 2.006 \\
\hline $\mathrm{HMBA}-\mathrm{Cr}^{v}$ & ref. 10 & 341 & 69.31 & 69.74 & 1.980 \\
\hline $\mathrm{EHBA}-\mathrm{Cr}^{\vee}$ & ref. 10 & 369 & 69.26 & 69.68 & 1.979 \\
\hline
\end{tabular}




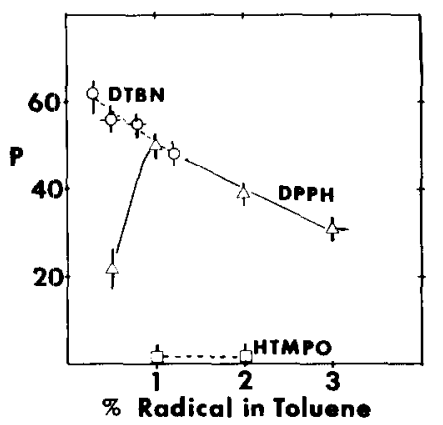

Fig. 1. The polarization in toluene is given as a function of the concentration of free radical used. The curves are drawn to guide the eye. Concentrations are expressed in percent by weight.

mum polarizations for positive and negative enhancements, and the relaxation time at $0.5 \mathrm{~K}$. In general we have considered these tests to be a first pass thru a multi-parameter space. The method of preparation might be varied next to further increase the polarizations.

Since the polarizations obtained in this study were only $\approx 50 \%$ we must address the question of how radiation resistant a material must be to be "better" than the standard alcohol targets. Thus we would like to assign a figure of merit to each material given its maximum polarization $P_{0}$ and characteristic flux $\Phi_{\mathrm{A}}$. Polarized target experiments typically measure some asymmetry

$A=\frac{N_{+}-N_{-}}{P\left(N_{+}+N_{-}\right)}$,

where $N_{+}\left(N_{-}\right)$is the number of events with the target polarized in the $+(-)$ direction, and $P$ is the target polarization. Experimentally we might define the "best" target material available to be the one that minimizes the error $\mathrm{d} A$ on the measurement of $A$ in a given period of time. For $N_{+} \simeq N_{-}=N$

$\mathrm{d} A=\frac{1}{\langle P\rangle \sqrt{ }(2 N)}$,

where $\langle P\rangle$ is the average target polarization during the period the $2 N$ events were collected. The quantity $\mathrm{d} A$ will differ for different substances since (a) the maximum polarizations differ thereby affecting $\langle P\rangle$, and (b) the characteristic fluxes $\Phi_{\mathrm{A}}$ differ affecting both $\langle P\rangle$ and $N$. The $N$ dependence arises from the time lost annealling and changing targets.

TABLI: 3

Summary of individual measurements.

\begin{tabular}{|c|c|c|c|c|c|c|}
\hline Radical & Solvent & $\%$ & Vacuum & $P^{+}$ & $P^{-}$ & $T_{\ln }(\mathrm{m})$ \\
\hline \multirow[t]{10}{*}{ DPPH } & Toluene & 0.5 & $\mathrm{Y}$ & $\sim 22$ & - & $\sim 340$ \\
\hline & & 1.0 & $\mathrm{Y}$ & $49 \pm 3$ & $50 \pm 3$ & $85 \pm 28$ \\
\hline & & 2.0 & $\mathrm{Y}$ & $40 \pm 3$ & $37 \pm 3$ & $64 \pm 7$ \\
\hline & & 3.0 & $\mathrm{Y}$ & $32 \pm 3$ & $29 \pm 3$ & $33 \pm 7$ \\
\hline & Mesitylene & 2.0 & $\mathrm{Y}$ & $17 \pm 4$ & - & $45 \pm 10$ \\
\hline & Ethylbenzene & 2.0 & $\mathrm{Y}$ & $37 \pm 3$ & $35 \pm 3$ & $48 \pm 10$ \\
\hline & Butyl ether & 1 (sat) & - & $\sim 44$ & $>32$ & $390 \pm 160$ \\
\hline & Ethyl formate & 2.0 & $N$ & $38 \pm 3$ & - & $74 \pm 6$ \\
\hline & Amyl acetate & 1.0 & Y & $\sim 45$ & - & $265 \pm 140$ \\
\hline & Benzyl alcohol & 2.0 & - & -10 & - & - \\
\hline \multirow[t]{9}{*}{ DTBN } & Toluene & 0.3 & $\mathrm{~N}$ & $65 \pm 5$ & $58 \pm 5$ & $\sim 320$ \\
\hline & & 0.5 & - & $57 \pm 3$ & $54 \pm 3$ & $142 \pm 20$ \\
\hline & & 0.8 & - & $55 \pm 3$ & $54 \pm 3$ & $79 \pm 25$ \\
\hline & & 1.2 & $\mathrm{Y}$ & $49 \pm 3$ & $46 \pm 3$ & $74 \pm 18$ \\
\hline & Butyl ether & 0.5 & $\mathrm{~N}$ & $\sim 40$ & - & $370 \pm 240$ \\
\hline & Ethyl formate & 0.5 & $\mathrm{~N}$ & $\sim 3$ & - & - \\
\hline & Amyl acetate & 0.5 & $\mathrm{~N}$ & $44 \pm 3$ & $42 \pm 3$ & $203 \pm 72$ \\
\hline & n-pentanol & 0.5 & $\mathrm{~N}$ & $48 \pm 3$ & $42 \pm 3$ & $100 \pm 11$ \\
\hline & Benzyl alcohol & 0.5 & $\mathrm{~N}$ & $40 \pm 4$ & $54 \pm 4$ & $125 \pm 15$ \\
\hline \multirow[t]{2}{*}{ HMBA-Crv } & n-pentanol & 1.8 & - & $51 \pm 3$ & $59 \pm 3$ & $165 \pm 30$ \\
\hline & & 5.3 (sat) & $\mathrm{Y}$ & $50 \pm 3$ & $58 \pm 3$ & $103 \pm 28$ \\
\hline $\mathrm{EHBA}-\mathrm{Cr}^{2}$ & n-pentanol & 2.0 & $\mathrm{Y}$ & $61 \pm 3$ & $63 \pm 3$ & $244 \pm 60$ \\
\hline \multirow[t]{3}{*}{$\mathrm{H} T \mathrm{MPO}$} & Toluene & 1.0 & $\mathrm{Y}$ & $\sim 2$ & - & - \\
\hline & & 2.0 & $\mathrm{Y}$ & $\sim 2$ & - & - \\
\hline & Butyl ether & 2.0 & $N$ & $13 \pm 4$ & $9 \pm 4$ & $18 \pm 6$ \\
\hline
\end{tabular}




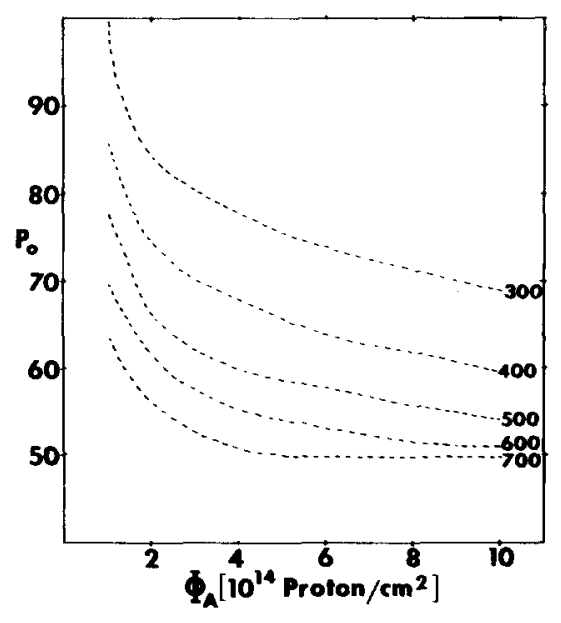

Fig. 2. The maximum polarization required in a new material is shown as a function of its characteristic radiation flux constant. The numbers by the curves refer to the $K$ values, which specify the numbers of events collected per $10^{14}$ particles $/ \mathrm{cm}^{2}$.

We have written a computer program to calculate for a given flux constant $\Phi_{\mathrm{A}}$ the maximum initial polarization $P_{0}$ necessary to achieve a $2 \% \mathrm{er}$ ror on $A$ in a seven day period. We have assumed a beam intensity of $10^{10}$ protons $/ \mathrm{s}$ over a $4 \mathrm{~cm}^{2}$ effective area. The polarization was assumed to fall off with radiation damage according to eq. (1). In addition the initial polarization following annealling was assumed to be related to the starting undamaged polarization $P_{0}$ in the same manner found for ethanediol, namely ${ }^{2}$ )

$P_{0}($ ann $)=P_{0}\left[\frac{P_{\mathrm{a}}}{P_{0}}+\left(1-\frac{P_{\mathrm{a}}}{P_{0}}\right) \mathrm{e}^{-\Phi / \Phi_{\mathrm{NA}}}\right]$,

where $P_{\mathrm{a}} / P_{0} \simeq 0.8$ and $\Phi_{\mathrm{NA}} \simeq 10^{14}$ protons $/ \mathrm{cm}^{2}$. The program optimized the number of target changes and anneals for each combination of $\Phi_{\mathrm{A}}$ and $P_{0}$. Anneals were assumed to require $90 \mathrm{~min}$ and target changes and accompanying thermal measurements $8 \mathrm{~h}$. It was further assumed to be impractical to anneal more than once every $8 \mathrm{~h}$ or change targets more than once each day.

The results are shown in fig. 2. The exact relationship between $P_{0}$ and $\Phi_{\mathrm{A}}$ depends on the experimental parameter $K$ which gives the number of events per $10^{14}$ protons $/ \mathrm{cm}^{2}$ on the target. The parameter $K$ essentially measures how fast events are collected compared to the rate the target is damaged. For large cross section processes the curve quickly flattens out and there is little advantage in using high $\Phi_{\mathrm{A}}$ materials. New materials will be most useful in measurements of asymmetries in small cross section processes. As a practical example consider the $K=400$ curve. Present diol targets $\left(\Phi_{\mathrm{A}} \simeq 2\right)$ can easily achieve the initial polarization of $74 \%$ required to make a $2 \%$ measurement of $A$ in one week. A new material with $\Phi_{\mathrm{A}}=10$ would only require an initial polarization of $59 \%$ to acheive the same precision. In addition a $\Phi_{\mathrm{A}}=10$ target only requires four changes and seven anneals during the week while the $\Phi_{\mathrm{A}}=2$ target requires four changes and fourteen anneals.

Several of the target materials investigated acheived polarizations greater than $50 \%$. The two best possibilities at this point appear to be toluene with $0.5 \%$ DTBN and n-pentanol with $2.0 \%$ EHBA-Crv ${ }^{v}$. These materials may be preferable to the diols under certain experimental conditions and should be candidates for further study.

I would like to thank the authors of ref. I for their assistance during the collection of data for this paper. I would also like to thank Drs. D. Hill of Argonne National Laboratory and M. Krumpolc and $J$. Rocek of the University of Illinois at Chicago Circle for providing the hydroxy acid-Cr. samples for these tests.

\section{References}

1) D. Crabb, R. Fernow, P. Hansen, A. Krisch, A. Salthouse, B. Sandler, K. Terwilliger, J. O'Fallon, E. Crosbie, L. Ratner and P. Schultz, Phys. Rev. Lett. 41 (1978) 1257.

2) R. Fernow, Nucl. Instr. and Meth. 148 (1978) 311.

3) J. Spinks and R. Woods, An introduction to radiation chemistry (J. Wiley, New York, 1964).

4) V. Voevodskii and Y. Molin, Rad. Res. 17 (1962) 366.

5) R. Alger et al., J. Chem. Phys. 30 (1959) 695.

6) Low solubility is defined as less than $0.5 \%$ for DPPH and PX and less than $1 \%$ for the $\mathrm{Cr}^{v}$ complexes

7) Although a polarization of $62 \%$ was achieved for this case (see table 3 ) the associated relaxation time was very long making this an impractical combination.

8) G. Hartmann et al., Proc. 2nd Int. Conf. on Polarized targets (ed. G. Shapiro; LBL 500, UC-34 Physics, 1972) p. 289. This is the average polarization in pure n-pentanol. Higher polarizations can be achieved by the addition of water but this may have a deleterious effect on the radiation resistance.

9) D. Hill et al., Nucl. Instr. and Meth. 150 (1978) 331. A closely related compound EHBA-Crv, Sodium bis(2-ethyl-2hydroxy-butyrato) oxochromate (V) monohydrate, was used for one of the trials as indicated in table 3.

10) M. Krumpolc and J. Rocek, J. Am. Chem. Soc. 98 (1976) 872. 